\title{
109. Impulse Transmission in Cat Lateral Geniculate and So-called Deep Sleep Wave
}

\author{
By Kitsuya Iwama and Hisatoshi Sakakura \\ Institute of Higher Nervous Activity, Osaka University \\ Medical School, Osaka \\ (Comm. by Yas Kuno, M.J.A., June 12, 1965)
}

In 1963 Brooks and Bizzi ${ }^{1)}$ made an interesting finding in the freely behaving cats that when the animals entered the phase of deep sleep (paradoxical sleep), there appeared a peculiar electrical activity (a burst of 3-4 waves of uniform sizes at 8/sec) in the lateral geniculate body (LGB), the oculomotor nucleus and the pontine reticular formation. Since this type of activity was observed only during deep sleep, these authors called it "deep sleep wave" (DSW). After having confirmed their findings, we attempted to know the effects of the DSW upon the impulse transmission in the LGB.

The experimental materials were exclusively the cats. They carried permanently implanted electrodes in the sensorimotor cortex, the LGB and the optic tract (OT) at the optic chiasma. Some cats had implanted electrodes near the ridge of the orbit, through which eye movements were recorded. Also we inserted needle electrodes into the posterior neck muscles in order to monitor their EMG activities.

The signs of deep sleep are well known. They are low voltage fast EEG activities in the sensorimotor cortex, a complete silence of the neck muscle EMG, rapid eye movements and others. When these signs were observed in behaviorally sleeping cats, it was always observed that the background LGB activity contained a frequent burst of the DSW. A typical example of the DSW will be seen in a vertically continuous record of the LGB activity of Fig. 1, right.

During deep sleep we stimulated the optic chiasma with a single, brief electric pulse and recorded the response to it from the LGB. The response appeared with essentially the same pattern as described by Vastola. ${ }^{2}$ Fig. 1 mounts several examples of the response displayed with a high sweep speed. They consisted of two components, the first being a positive-negative-positive spike and the second a negative wave of a relatively long duration. According to the widely accepted interpretation, the first triphasic spike is the activity of the OT terminals and the second monophasic wave the discharges of the transsynaptically activated LGB neurons. While continuing the 
chiasmatic stimulation during deep sleep, we noted that the postsynaptic component of the evoked LGB response tended to increase when the stimulation timed with the spontaneous DSW. In order to make this point clearer, a statistical examination was carried out. We sampled the total of 50 responses evoked at a rate of $0.5 / \mathrm{sec}$ during a single episode of deep sleep. Among them 11 responses were found to collide with the spontaneous DSW. Using the all responses obtained we constructed an amplitude histogram of the postsynaptic discharges and found that a range of extremely high amplitudes of the histogram was occupied by the responses which were obtained under the influence of the DSW (Fig. 1, right). It is thus concluded that the DSW is effective to facilitate the synaptic transmission in the LGB.

The action of the DSW, however, is not limited to the facilitation at the postsynaptic level. When one scrutinizes the records of the evoked LGB response during deep sleep, one can see that the amplitude of the presynaptic component also fluctuates depending upon whether or not the background LGB activity contains the DSW; there is a tendency for the presynaptic component to decrease when the response

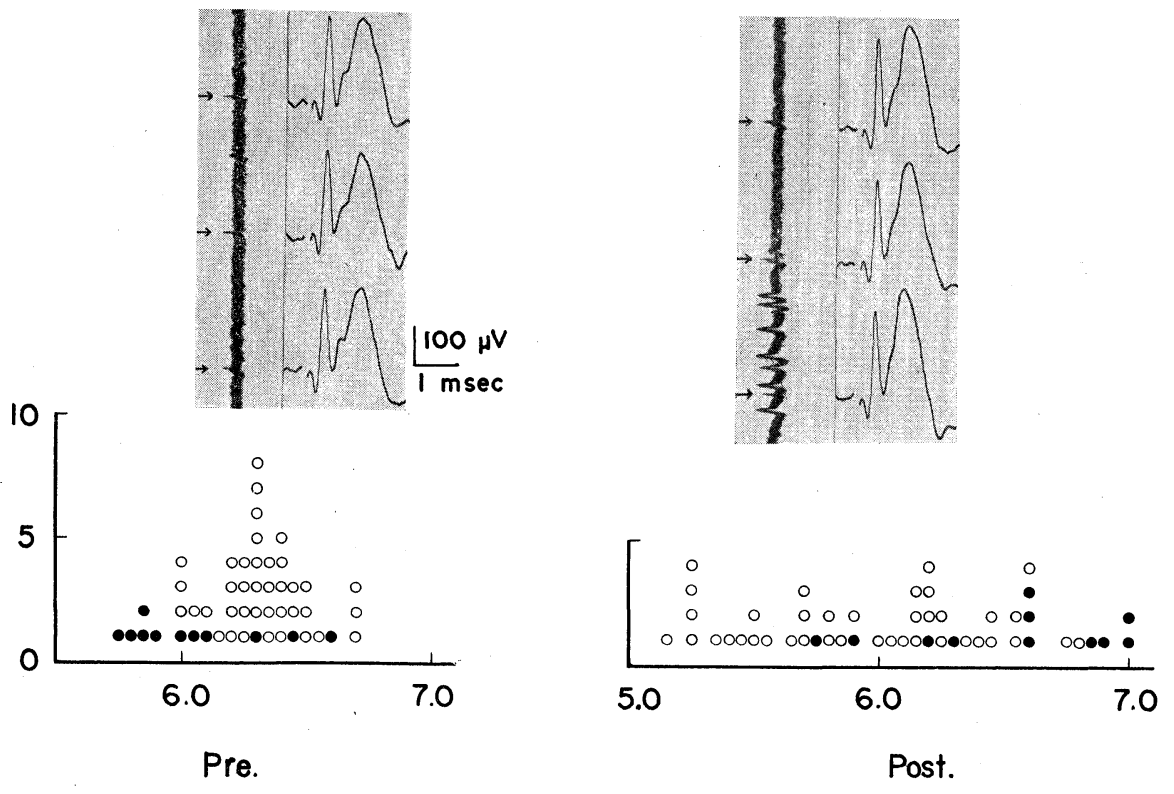

Fig. 1. Evoked LGB responses and amplitude histograms of presynaptic and postsynaptic components. Evoked responses were obtained with continuous display of the background LGB activity. In the right group of the specimen records, DSW is seen in a lower half of the vertically continuous record. Arrows indicate the chiasmatic stimulation. Responses obtained in the presence of the DSW are marked with filled circles in the histograms. 
is evoked timing with the DSW. A statistical evaluation of this fact will be made with the amplitude histogram of the presynaptic component which was constructed with the same data as for the histogram of the postsynaptic component (Fig. 1, left). According to our previous experiment ${ }^{3}$ a suppression of the presynaptic component of the evoked LGB response signifies that there occurs a depolarization at the OT terminals resulting a reduction of the action potentials therefrom (presynaptic inhibition).

That the DSW induces the presynaptic inhibition on the OT terminals could be demonstrated in a different way. We applied a brief electric pulse to the OT terminals through the LGB electrodes and recorded the antidromically conducted OT response from the optic chiasma. If there might be a depolarization in the OT terminals in association with the DSW, more fibers would be excited to a stimulus of a given intensity so that the antidromic OT response would be larger as compared with that elicited in the abscence of the DSW $\left(\mathrm{Wall}^{4}\right)$. As shown by the specimen records in Fig. 2, the above expectation was almost completely fulfilled. The amplitude histogram of the antidromic OT response clearly shows that most of the responses obtained in association with the DSW are accumulated in a range of the extremely high amplitudes.

Discussion. A functional significance of the DSW remains unclear. Since it is recordable from the LGB and the oculomotor nucleus and is associated with rapid eye movements, it would be likely that the DSW might closely be related to the visual function. Supporting this view, we have established that the synaptic trans-
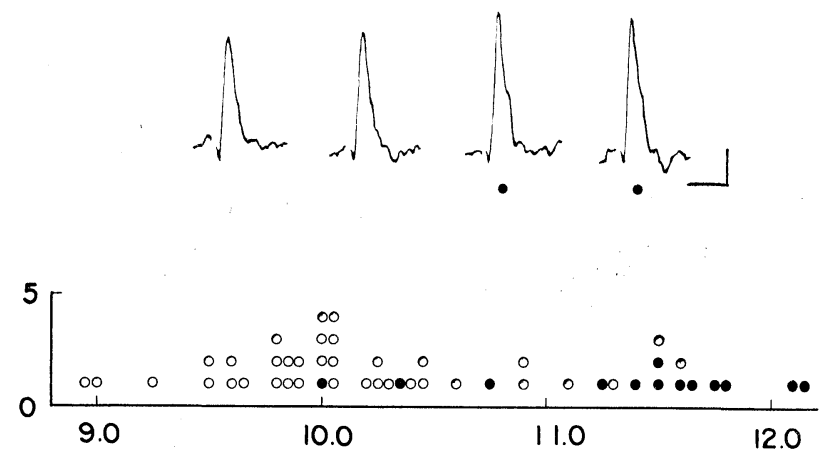

Fig. 2. Antidromic OT responses and amplitude histogram. Among four responses illustrated, the right two were obtained during the DSW and the left two in the abscence of DSW. The total of 49 responses, obtained during a single episode of deep sleep, were used for the histogram where the responses related to DSW are marked with filled circles. 
mission in the LGB is profoundly modified by the DSW. But the modified condition of the synaptic transmission seems rather peculiar; there occurs inhibition on the presynaptic side and facilitation on the postsynaptic side. The functional significance of such modified condition is difficult to understand. However, as one of the possible consequences, one can imagine the following. First of all, since there is an inhibition in the input side of the LGB in association with the DSW, some impulses arising from the retina may be stopped from reaching the visual cortex when they collide with the DSW. This may suggest that the DSW may serve to maintain the phase of deep sleep by minimizing a disturbance due to visual impulses. However, since the presynaptic inhibition due to the DSW is not absolutely powerful, some impulses may escape the inhibition and reach the visual cortex after having been amplified at the postsynaptic level of the LGB. These unusually amplified impulses may be interpreted by the cortex as having a great biological significance. We suppose that such situation might be responsible for dreaming which is known to occur mainly during deep sleep (Dement and Kleitman ${ }^{\text {s) }}$; Jouvet and Jouvet $^{6)}$ ).

\section{References}

1) Brooks, D. C., and Bizzi, E.: Arch. ital. Biol., 101, 648 (1963).

2) Vastola, E. F.: J. Neurophysiol., 20, 167 (1957).

3) Iwama, K., Sakakura, H., and Kasamatsu, T. : Jap. J. Physiol., 15, 310 (1965).

4) Wall, P. D.: J. Physiol., 142, 1 (1958).

5) Dement, W., and Kleitman, N.: Electroenceph. clin. Neurophysiol., 9, 673 (1957).

6) Jouvet, M., and Jouvet, D.: Electroenceph. clin. Neurophysiol., Suppl. 24, 133 (1963). 